\title{
Psicooncología
}

ISSN: 1696-7240

\section{La revelación del diagnóstico en oncología: una investigación transcultural Brasil-España}

\author{
Elisa Kern de Castro하 Franciele Peloso ${ }^{2}$; Luísa Vital ${ }^{3}$; Fernanda Bittencourt Romeiro \\ Lourdes Moro Gutiérrez ${ }^{5}$; Marta González Fernández-Conde ${ }^{6}$
}

Recibido: 29 de septiembre de 2017 / Aceptado: 23 de enero de 2018

Resumen. Objetivo: examinar aspectos transculturales de la revelación del diagnóstico de cáncer en pacientes brasileños y españoles. Método: Se realizó una investigación cualitativa en la que fueron entrevistados 28 pacientes en quimioterapia (14 brasileños y 14 españoles). El análisis de contenido identificó tres ejes temáticos: 1) Cómo fue revelado el diagnóstico; 2) Contexto en que el diagnóstico fue revelado y 3) Quién reveló el diagnóstico. Resultados: existen particularidades en la manera de revelar el diagnostico entre los dos países. En Brasil aún ocurre que el enfermo puede ser derivado a un oncólogo sin una explicación satisfactoria previa sobre su estado de salud. Aunque en los dos países existan relatos de comunicación de diagnóstico en el contexto apropiado (consulta), también ha aparecido revelación de manera informal (por teléfono) sin un ambiente adecuado para que el paciente sea acogido y tenga posibilidad de expresar sus miedos y sus dudas. Conclusión: es necesario invertir en la formación profesional para mejorar la habilidad de comunicación y educación en salud para tratar pacientes con cáncer.

Palabras clave: cáncer; comunicación; relación médico-paciente.

\section{[en] Cancer disclosure: a cross-cultural research Brazil-Spain}

\footnotetext{
Abstract. Objective: The aim of this study was to examine cross-cultural aspects of the disclosure of cancer diagnosis in Brazilian and Spanish patients. Method: A qualitative research was carried out in which 28 chemotherapy patients (14 Brazilian and 14 Spanish) were interviewed. Content analysis identified three thematic categories: 1) How the diagnosis was disclosured; 2) Context in which the diagnosis was disclosured; 3) Who disclosured the diagnosis. Results: Particularities in the diagnosis disclosure between the two countries was found. In Brazil, it still happens that patient can be referred to an oncologist without a satisfactory explanation about his health status. Although there are reports of diagnosis disclosure in the appropriate context (consultation), also there are reports informal disclosure

1 Elisa Kern de Castro. Universidade do Vale do Rio dos Sinos, São Leopoldo, Brasil.

E-mail: elisakc@unisinos.br

2 Franciele Peloso. Universidade do Vale do Rio dos Sinos, São Leopoldo, Brasil.

E-mail: francielepeloso@outlok.com

3 Luísa Vital. Universidade do Vale do Rio dos Sinos, São Leopoldo, Brasil.

E-mail: luisavital1@hotmail.com

4 Fernanda Bittencourt Romeiro. Universidade do Vale do Rio dos Sinos, São Leopoldo, Brasil.

E-mail: romeiro.fernanda@hotmail.com

5 Lourdes Moro Gutiérrez. Universidad de Salamanca, Salamanca, España.

E-mail: moro@usal.es

6 Marta González Fernández-Conde.Fundación Hospital General Santísima Trinidad, Salamanca, España.

E-mail: quimioterapia@fhgst.es

* Dirección de correspondencia: Elisa Kern de Castro-Avenida Unisinos 950, Bairro Cristo Rei, São Leopoldo, RS, Brasil.

E-mail: elisakc@unisinos.br
} 
(by telephone), without an adequate environment for the patient to be welcomed and able to express their fears and doubts. Conclusion: It is necessary to improve the professional training to improve communication skills and health education to treat cancer patients.

Keywords: cancer; communication; physician-patient relationship.

Sumario. 1. Introducción 2. Método 2.1. Participantes 2.2. Instrumentos 2.3. Procedimientos para recogida de dados y aspectos éticos 2.4. Análisis de los datos 3. Resultados y discusión 4. Consideraciones finales 5. Referencias bibliográficas.

Cómo citar: Kern de Castro E, Peloso F, Vital L, Bittencourt Romeiro F, Moro Gutiérrez L, González Fernández-Conde M. La revelación del diagnóstico en oncología: una investigación transcultural Brasil-España. Psicooncología 2018;15:119-132. Doi: 10.5209/PSIC.59181.

\section{Introducción}

Comunicar el diagnóstico de cáncer a un paciente es un desafío para cualquier profesional sanitario. En el pasado, revelar el diagnóstico de cáncer era considerado un acto inhumano y perjudicial para el enfermo. Hoy en día, la importancia del cómo y cuándoun diagnóstico de cáncerdebe ser reveladoesuno delos aspectos más destacados en investigación en psicooncología ${ }^{(1,2)}$. Es fundamental que en esa consulta tanto el paciente como sus acompañantes puedan expresar y hacer preguntas sobre sus dudas y temores a la vez que establecen, junto a su médico, el plan de tratamiento a seguir.

En el contexto de la psicooncología, las habilidades de comunicación del médico y la percepción del paciente sobre su relación con los profesionales, son aspectos esenciales para una comunicación con éxito. Las metas de esta comunicación están relacionadas con el manejo de cuestiones médicas como la revelación del diagnóstico y la información sobre la enfermedad y el tratamiento y con las respuestas del propio afectado relacionadas con la comprensión de la enfermedad y la satisfacción y bienestar por la información recibida. Para ello, una comunicación sincera y clara en el momento del diagnóstico permite al paciente expresar sus sentimientos, dudas y preocupaciones de cara a comprender su estado de salud ${ }^{(3,4)}$. El uso de un lenguaje sencillo y el contacto visual son también elementos fundamentales en el momento de la comunicación ${ }^{(5)}$.

La información y el proceso de comunicación en el que se aporta se desarrollan en un contexto sociocultural concreto que está definido por las personas directamente implicadas y que tienen sus propias necesidades y expectativas. Se establece una relación entre personas que pertenecen a culturas diferentes, la del profesional y la del paciente y su familia, pero que tienen un objetivo común que es la curación del enfermo ${ }^{(6)}$. Ante la posibilidad de tener cáncer las personas reaccionan de diferente manera, unos buscan información y otros la evitan por miedo, dependiendo de factores situacionales, de personalidad y psicológicos. Médicos y profesionales de enfermería deben adaptar la información que proporcionan a las necesidades de los pacientes ${ }^{(7)}$.

Comunicar a una persona noticias como un diagnóstico de cáncer, explicar los tipos de tratamientos disponibles o informar sobre resultados de pruebas clínicas que indican recidiva y/o metástasis, deberían partir del conocimiento por parte de los profesionales de aspectos personales de los afectados que pueden influir en su aceptación ${ }^{(8)}$. En la información sobre los resultados de salud las personas pueden manifestar su preferencia por la comunicación cara a cara o por otros medios electrónicos. Sin embargo, la revelación del diagnóstico de cáncer requiere que en cualquier contexto sociocultural 
la comunicación sea cara a cara, considerando el posible impacto negativo de la noticia que puede necesitar un apoyo emocional inmediato por arte de los profesionales.

Las consecuencias que se derivan de un proceso de comunicación inadecuado afectan a la adaptación a la enfermedad y a la calidad de vida y pueden provocar ansiedad, depresión, desesperanza, sufrimiento y rechazo de las medidas terapéuticas ${ }^{(9)}$. La información es una herramienta fundamental para el paciente oncológico pues facilita su participación activa en la gestión de sus cuidados de salud, en el autocuidado y en la toma de decisiones ${ }^{(6,10)}$.

El análisis de los diferentes sistemas sanitarios de Brasil y España apunta que es más común en Brasil optar por planes privados de salud. En ambos casos, la mayor parte de los pacientes entrevistados conocen el diagnóstico a través de médicos no especialistas que les derivan al oncólogo. En el caso de Brasil debido a los tiempos de espera para conseguir cita para la consulta y las dificultades de acceso a exámenes clínicos específicos, un gran número de personas encuentra en los planes de salud privados la solución para estas esperas ${ }^{(11)}$. En el sistema sanitario español actual, la mayor parte de los pacientes oncológicos son tratados en el sistema público de salud, pero la muestra analizada en el artículo corresponde a pacientes de un hospital privado, el Hospital de a Santísima Trinidad de Salamanca. Aunque estos pacientes pertenecen a MUFACE (Mutualidad General de Funcionarios civiles del Estado) y su tratamiento no tiene ningún coste económico para ellos. El médico de familia puede ser el profesional que inicialmente detecte los problemas de salud y después de realizar las pruebas necesarias deriva a la persona al especialista necesario. En este sentido, Spiegel et al. (2010) ${ }^{(12)}$ en un estudio realizado en Austria analizan la calidad de la comunicación entre los diferentes profesionales de la salud que intervienen en el tratamiento contra el cáncer y señalan que los enfermos conceden mucha importancia a la información aportada por su médico de familia y consideran que la información que estos profesionales reciben de los profesionales de los centros hospitalarios es insuficiente. Los autores hipotetizan que esto mismo puede extrapolarse a otros sistemas sanitarios europeos. Además apuntan que la sospecha inicial de que una persona pueda tener cáncer en el $42,1 \%$ de los casos proviene de un médico fuera de los centros hospitalarios.

Además de las cuestiones culturales relacionadas con el contexto geográfico y de organización del sistema sanitario, otro aspecto cultural importante en las últimas décadas ha sido la inclusión de otros profesionales del área asistencial en el equipo de salud ${ }^{(13,14)}$. La inclusión de profesionales de las áreas de nutrición, psicología y fisioterapia además de medicina y enfermería ha tenido repercusiones en la relación médico-paciente. La diversidad de profesionales que incluye el equipo sanitario facilita y mejora el proceso de comunicación del cáncer. El paciente pasa a recibir información de la enfermedad sobre numerosos aspectos, por ejemplo, el nutricionista le informa sobre la dieta más adecuada para paliar algunos de los efectos secundarios derivados de los tratamientos y el psicólogo le ayuda en su el proceso de aceptación de la enfermedad y en su expresión emocional. Todo ello con el objetivo de conseguir la mayor calidad de vida y bienestar posible para el paciente y su entorno ${ }^{(15,16)}$. El trabajo interdisciplinar es el foco de varias discusiones en el área de la salud, sin embargo, en la práctica hacen falta investigaciones que examinen su impacto en la relación profesional-paciente, especialmente en el contexto de la oncología. Así, el objetivo del presente artículo es examinar cualitativamente aspectos contextuales y transculturales que permean la relación entre el paciente con cáncer y el equipo sanitario que le atiende, en lo que se refiere a la revelación del diagnóstico en un grupo de pacientes brasileños y españoles. 


\section{Método}

Diseño: Cualitativo exploratorio y de comparación transcultural (Brasil-España). El abordaje cualitativo, según Hayes (2000), busca comprender el significado de las conductas humanas a partir de la experiencia subjetiva de las personas. Por otro lado, los estudios transculturales pueden ser exploratorios, una vez que no se incluyen variables contextuales y se utilizan explicaciones a posteriori para dar significado a las diferencias culturales ${ }^{(17)}$.

\subsection{Participantes}

Participaron 28 pacientes oncológicos adultos en tratamiento de quimioterapia para el cáncer en dos hospitales: el primer ubicado en Porto Alegre (Brasil), y el otro en la ciudad de Salamanca (España). La elección de los participantes fue por conveniencia entre aquellos que estaban en el hospital para la realización de quimioterapia en el momento de la entrevista. La recogida de los datos ha seguido hasta que se constató que las informaciones no traían nuevos elementos para la teorización a respecto del objeto de la investigación ${ }^{(18)}$.

En Brasil, participaron 14 pacientes, seis mujeres y ocho hombres, con edad media de 52,36 años $(\mathrm{DT}=3,10)$. Los datos sociodemográficos y clínicos de los participantes brasileños se encuentran en la Tabla 1:

Tabla 1. Descripción de los datos sociodemográficos y clínicos de los participantes brasileños $(\mathrm{n}=14)$.

Tratamiento

\begin{tabular}{cccccccccc}
\hline Paciente & Sexo & Edad & $\begin{array}{c}\text { Estado } \\
\text { Civil }\end{array}$ & Escolaridad & $\begin{array}{c}\text { Diagnóstico } \\
\text { CA }\end{array}$ & Metástase & C & R & Q \\
\hline 1 & M & 37 & Casada & Superior & Útero & Sí & No & No & Sí \\
2 & M & 64 & Soltera & Post-Grado & Intestino & Sí & Sí & No & Sí \\
3 & H & 70 & Casado & Superior & Cólon & Sí & Sí No & Sí \\
4 & H & 58 & Casado & ESO & Cólon & No & No & No & Sí \\
5 & M & 44 & Casada & Superior & $\begin{array}{c}\text { Estómago/ } \\
\text { Esofago }\end{array}$ & No & Sí & Sí & Sí \\
6 & M & 65 & Casada & ESO & Ovário & No & Sí & No & Sí \\
7 & H & 52 & Casado & Superior & Intestino & No & No & No & Sí \\
8 & M & 45 & Separada & Primaria & Intestino/ & Sí & No & No & Sí \\
9 & H & 66 & Casado & ESO & Intestino & Sí & Sí & Sí & Sí \\
10 & H & 65 & Casado & ESO & Intestino & Sí & No & No & Sí \\
11 & H & 54 & Casado & ESO & Piel/ & No & No & No & Sí \\
12 & H & 33 & Casado & Superior & Testículo & No & Sí No & Sí́ \\
13 & H & 30 & Soltero & Superior & Recto & Sí & Sí & Sí & Sí \\
14 & F & 50 & Soltera & Superior & Útero & No & Sí & No & Sí \\
\hline
\end{tabular}

Nota. M-Mujer; H-Hombre.. C - Cirugía; R - Radioterapia; Q - Quimioterapia. 
En España, también participaron 14 pacientes, diez mujeres y cuatro hombres, con edad media de 51,90 años ( $\mathrm{DT}=10,70)$. Los datos sociodemográficos y clínicos de los participantes españoles están en la Tabla 2:

Tabla 2. Descripción de los datos sociodemográficos y clínicos de los participantes españoles $(n=14)$.

Tratamiento

\begin{tabular}{cccccccccc}
\hline Paciente & Sexo & Idade & $\begin{array}{c}\text { Estado } \\
\text { civil }\end{array}$ & Escolaridade & $\begin{array}{c}\text { Diagnóstico } \\
\text { CA }\end{array}$ & Metástase & C & R & Q \\
\hline 1 & H & 35 & Casado & Superior & Testículo & No & Sí & No & Sí \\
2 & H & 64 & Casado & ESO & Pulmón & No & No & No & Sí \\
3 & M & 61 & Casada & Superior & Pulmón & No & No & No & Sí \\
4 & M & 43 & Casada & Superior & Mama & No & Sí & Sí & Sí \\
5 & M & 53 & Casada & Primaria & Mama & No & Sí & No & Sí \\
6 & H & 52 & Casado & Superior & Cólon & No & Sí & No & Sí \\
7 & M & 46 & Soltera & Superior & Ovário & No & Sí & No & Sí \\
8 & M & 45 & Casada & Superior & Mama & No & Sí & No & Sí \\
9 & M & 59 & Soltera & Superior & Mama & Sí & Sí & Sí & Sí \\
10 & M & 48 & Casada & Superior & Mama & Sí & Sí & Sí & Sí \\
11 & M & 32 & Casada & Superior & Mama & No & Sí & Sí & Sí \\
12 & H & 65 & Casada & Superior & Cólon & No & Sí & No & Sí \\
13 & M & 66 & Viudo & Superior & Mama & No & Sí & No & Sí \\
14 & M & 49 & Casada & Superior & Mama & No & Sí & Sí & Sí \\
\hline
\end{tabular}

Nota. M-Mujer; H-Hombre. C - Cirugía; R - Radioterapia; Q - Quimioterapia.

\subsection{Instrumentos}

- Datos sociodemográficos y clínicos: contiene informaciones para caracterizar a los participantes, como edad, sexo, escolaridad, diagnóstico, fecha del diagnóstico, tipo de tratamiento realizado.

- Entrevista semi-estructurada sobre la relación profesional sanitario-paciente: fueron creadas preguntas sobre las siguientes temáticas: vinculo profesionalpaciente, diagnóstico y tratamiento oncológico y sufrimiento psicológico. En la presente investigación, se analizó solamente los datos sobre la revelación del diagnóstico.

\subsection{Procedimientos para recogida de dados y aspectos éticos}

La recogida de los datos empezó después de la aprobación del proyecto de investigación por parte de Comisión de Ética en Investigación de la Universidad. En Brasil y en España, la invitación para participar y las aclaraciones sobre los objetivos de la investigación fueron realizados por el investigador en el hospital mientras el paciente hacía su tratamiento. Después de su aceptación para participar, todos firmaron el documento de Consentimiento. Las entrevistas se realizaron mientras el enfermo estaba recibiendo quimioterapia, controlando siempre su bienestar en cuanto 
a las condiciones y el contexto en el que se estaban realizando, para que estuviera lo más cómodo posible, cuidando también que otras personas no interfiriesen en ese momento. Los pacientes fueron invitados a participar después de que el equipo de enfermería confirmaba si el paciente tenía condiciones para contestar a la entrevista. La entrevista fue realizada por investigadores nativos en lengua portuguesa-brasileña (Brasil) y castellano (España), y fueron transcritas integralmente.

\subsection{Análisis de los datos}

En el presente artículo, fueron analizados solamente los contenidos relacionados con las preguntas sobre la revelación del diagnóstico. Se preguntó al paciente acerca de cómo le habían comunicado la noticia del diagnóstico de cáncer, quien le informó y qué aspectos cree que deberían haber sido diferentes o haberse tenido en cuenta en ese momento.

Los datos fueron analizados cualitativamente. Fue utilizado el protocolo COREQ (Consolidate Criteria for reporting qualitative research) ${ }^{(19)}$, un checklist de 32 ítems que busca garantizar que los criterios de calidad de un artículo científico cualitativo que utiliza entrevista o grupo focal sean cumplidos. Fue realizado análisis de contenido del material transcrito, con el foco en el tema de la revelación del diagnostico del cáncer. Los datos fueron analizados por dos investigadores, en tres etapas: 1) lectura inicial sin juicios; 2) análisis estructural y categorización del contenido; y 3) interpretación crítica y discusión ${ }^{(20)}$.

Después de creadas las categorías, los investigadores han entrenado dos jueces sobre el análisis de contenido y evaluación y categorización de los contenidos. Los jueces han categorizado los contenidos de manera independiente y de acuerdo con las categorías creadas. El índice kappa de concordancia entre los jueces ha sido de 0,84 , considerado muy bueno ${ }^{(21)}$.

\section{Resultados y discusión}

A partir del relato de los pacientes y del análisis de contenido, emergieron tres ejes temáticos: 1) Cómo fue revelado el diagnóstico (contenido de la información); 2) Contexto en que el diagnóstico ha sido revelado (dónde); 3) Quién ha revelado el diagnostico.

\section{Eje temático 1: Cómo fue revelado el diagnóstico}

El eje temático sobre cómo el diagnóstico fue revelado engloba respuestas sobre los diferentes contenidos informados por el médico en la consulta, además de cómo fuera de completa la información aportada por el médico en el momento de la comunicación. En este sentido, fueron identificadas tres categorías: a) revelación completa; b) revelación parcial; c) ausencia de revelación formal del diagnóstico.

En la Revelación completa, el diagnóstico de cáncer fue revelado al paciente de forma clara y con detalles sobre las características de la enfermedad y su tratamiento. La comunicación del médico fue respetuosa y aclaradora:

"Ya me ha dicho (el médico): mira, tu cáncer es así, va a funcionar así, vamos empezar hoy con la quimioterapia y va a ser asi desde ahora hacia delante. Tienes un $80 \%$ de posibilidad de quedarte sana en ese momento." (Paciente 1 - Brasil) 
"De forma clara, con optimismo por parte del médico, con gran interés y afecto. Me lo comunico el ginecólogo. Considero que fui informada de la forma más correcta y adecuada." (Paciente 4 - España)

En los testimonios de los pacientes se recoge que la revelación completa puede traer beneficios psicológicos al paciente, pues está asociada a la tranquilidad y claridad de lo que es necesario afrontar. Se constata también que el médico, al revelar el diagnóstico de manera clara, les da esperanza de un buen pronóstico, tanto en pacientes brasileños como españoles ${ }^{(22)}$. Además, la honestidad se muestra como una variable esencial en el momento de la revelación, sin falsas esperanzas sobre el futuro. Se sabe que una comunicación honesta y clara por parte del médico u otro profesional de la salud posibilita al paciente comprender mejor su enfermedad y sacar dudas sobre su tratamiento ${ }^{(4)}$. Así, una revelación completa se caracteriza por informaciones precisas sobre la enfermedad y de sensibilidad y empatía por parte del médico. Las informaciones y la sensibilidad y apoyo emocional que el profesional debe tener al comunicar el diagnóstico no aparecen de manera clara en los contenidos de los pacientes. Eso puede haber ocurrido porque los pacientes dan más importancia en ese momento a la información honesta sobre la enfermedad y el apoyo emocional es algo que está subyacente, como muestran otras investigaciones ${ }^{(5,23,24)}$.

"Me diagnosticaron muy rápido, he actuado con rapidez. Ha sido, así, tranquilo... Él (médico) me ha informado el resultado, me ha tranquilizado: "Mira, de los medios que tenemos hoy, estamos muy adelantados (en el tratamiento)." (Paciente 11 - Brasil).

"Yo creo que el médico ha sido firme y me ha dicho en el momento: tú tienes cáncer. Yo creo que eso es muy importante, porque si él empieza 'es posible que sea', entonces tú te quedas en duda, ¿será que tengo o no tengo? El hombre ha sido concreto, ha sido $100 \%$. El tío que te mira a los ojos y te dice la verdad." (Paciente 10 - Brasil).

"Me lo dijeron de forma escueta y sencilla, directa y simple, sin alarmismo". (Paciente 10-España)

La categoría Revelación parcial incluye contenidos en los que los pacientes han relatado que las informaciones aportadas por los médicos han sido incompletas. Esa información ha generado dudas, ansiedad y sufrimiento al paciente, que muchas veces se ha sentido incómodo para preguntar más detalles sobre su estado de salud:

"La persona que me hizo la ecografia me dijo así: 'tenemos un tumor alli'. No ha dicho si era benigno o maligno" (Paciente 2 - Brasil)

"Me dio mucha información a grandes rasgos para el futuro, aunque no me dio toda la información, mejor". (Paciente 8 - España)

"Me lo dijo (el diagnóstico) mi ginecólogo al recibir los resultados de las pruebas. Fue muy amable, aunque me explicó poco las cosas". (Paciente 14- España)

Además de ello, en esta categoría el testimonio de uno de los pacientes destaca la frialdad del profesional al revelarle el diagnóstico. Se aprecia que la comunicación técnica sin una preocupación sobre la salud y la vida del paciente le genera insatisfacción: 
"En el momento en que él (médico) me ha informado (el diagnóstico), yo le vi, así, muy insensible. Él parecía más preocupado con la cirugía que con mi bienestar" (Paciente 4-Brasil).

Las consecuencias que derivan de una revelación parcial pueden afectar de forma negativa en la vida del paciente, especialmente en lo que respecta a su comprensión y adaptación al cáncer y a su tratamiento. Además, trae consecuencias negativas para su salud mental, como ansiedad, depresión y desesperanza ${ }^{(9)}$.

La categoría Ausencia de revelación formal del diagnóstico de cáncer ha ocurrido en situaciones en las que el médico ha omitido los síntomas de la enfermedad o ha derivado al paciente al oncólogo o centro de salud sin informarlo sobre las hipótesis diagnósticas, refiriéndose solamente a un tratamiento complementario. La derivación a otro centro o profesional sin una conversación que le informe sobre la posibilidad de que pudiera tener cáncer genera intenso sufrimiento y levanta muchas dudas en el enfermo, pudiendo llevarle a pensar que su enfermedad es incurable o de difícil tratamiento $^{(9)}$. Está situación solamente se produce en los pacientes brasileños, no en los españoles, debido a las diferencias entre los sistemas sanitarios de ambos países. Por lo que solamente disponemos de testimonios de enfermos brasileños:

"Inicialmente yo he ido consultar en [nombre del pueblo], entonces un médico clínico me ha pedido una ecografía. Y, entonces, él me dijo que tenía que hacer un tratamiento, y que él no me podría tratar porque él no tenía condiciones para ello. '[Me dijo] que yo debería buscar un centro de referencia" (Paciente $9-$ Brasil).

"Me hicieron la cirugía y entonces, cuando fui mirar a los puntos, él [médico] me dijo que era necesario hacer un tratamiento complementario. Entonces él mismo me indicó otro médico, él mismo que marcó la cita. Y yo he venido hasta aquí." (Paciente 7 - Brasil).

A pesar de que las malas noticias son frecuentes en el contexto de la oncología, muchas veces los médicos de familia no se sienten preparados para ese momento, pues revelar un diagnóstico de cáncer implica saber controlar una difícil situación emocional al tener que explicar una enfermedad tradicionalmente asociada a muerte $^{(1,4)}$. Por ello, una manera de poder evitar esta situación delicada y difícil es derivar al enfermo a otro profesional y/o a las instituciones especializadas para que se encargue de comunicarle el diagnóstico.

“Él (medico) no dijo nada, dijo solo que yo no tenía nada muy grave. Que yo no tenía que preocuparme, que luego todo iba a pasar, y no pasó... sólo quedó cada vez peor (Paciente 9 - Brasil)

"Directamente (el médico) no me lo dijo nadie (sobre o diagnóstico de cáncer) (Paciente 11- España)

Para los médicos y profesionales de salud que tienen dificultades a la hora de comunicar el diagnóstico al paciente existen protocolos específicos, validados y reconocidos, que facilitan esta labor, como el protocolo SPIKES ${ }^{(1)}$. Este protocolo está dividido en seis pasos e incluye las técnicas que el profesional deberá seguir para dar una información completa y con sensibilidad a su paciente. Se espera que 
en la formación de los futuros profesionales sanitarios haya aprendizajes sobre cómo dar malas noticias a los pacientes, pero de momento, la formación aún carece de entrenamiento en el área de las relaciones humanas y de la comunicación ${ }^{(25)}$.

Eje Temático 2: Contexto en que el diagnóstico ha sido revelado

Con respecto al contexto en que el diagnostico ha sido revelado, se identificaron dos categorías a través de los relatos de los pacientes: diagnóstico revelado durante la consulta médica y otros. La categoría Consulta incluye las respuestas más comunes sobre el diagnóstico y las posibilidades de tratamiento, que surgen al revelar el diagnóstico, en ese espacio de privacidad entre el médico y el enfermo y su familia ${ }^{(26)}$.

"Me lo dijo la ginecóloga en su consulta." (Paciente 8 - España)

"Me lo dijeron en la consulta después de cinco meses de tratamiento equivocado". (Paciente 3 - España)

Se aprecia que el paciente se siente más cómodo al recibir el diagnóstico en el ambiente de la consulta médica, pues cuenta con la presencia y apoyo del médico y la comunicación ocurre cara a cara, lo que facilita la aclaración de dudas y reduce el impacto negativo del diagnóstico ${ }^{(8)}$.

"El diagnóstico me ha sido dicho por el doctor del hospital [nombre del hospital]. La manera que yo la he recibido fue muy tranquila" (Paciente 11 - Brasil)

"Fui al médico de cabecera de toda la vida. Estoy muy contenta con cómo me dijeron todo". (Paciente 5 - España)

"Cuando fui a la consulta con los resultados de las pruebas los vio y me dijo que lo que tenía era malo y que habría que operar. No estoy descontento con cómo me lo dijeron. Me parece bien como se hizo." (Paciente 6 - España)

En la categoría Otros, el diagnóstico ha sido revelado por el paciente en un ambiente fuera del contexto de la consulta médica tradicional. En general, ese diagnóstico ha sido dado de manera informal durante la realización de exámenes de imagen.

"El momento en que supe que era un tumor fue durante el examen de ecografia" (Paciente 2 - Brasil)

"Después de recibir los resultados de una analítica y de una ecografia, me lo dijeron." (Paciente 2 - España)

En el testimonio de un paciente brasileño, el diagnostico le fue comunicado durante una conversación por teléfono. El médico llamó al paciente y le comunicó el diagnóstico sin ningún tipo de consideración emocional:

"En el inicio de la tarde ella me llamó (médica). Ella me dijo: 'sí, realmente tú estás con neumonía, con un pequeño derrame en la pleura, pero también tienes cáncer'. Yo creo que eso ha sido muy malo, ella no ha tenido ninguna sutileza. Fue una manera muy poco delicada, me ha tirado una piedra" (Paciente 13 Brasil). 
La investigación de Neto et al. (2013) ${ }^{(27)}$ mostró que, en general, los pacientes se consideran poco preparados para recibir la noticia del diagnóstico por lo que es fundamental que el médico tenga las habilidades de comunicación necesarias para hacerlo de una manera amigable y tranquila. Las percepciones negativas en relación a la comunicación de los profesionales estaban relacionadas con la transmisión de la información de manera indiferente, sin una preocupación con el bienestar del paciente. Por lo tanto, es importante que la comunicación de malas noticias en salud sea aportada por personas que tengan habilidades de comunicación en las que la humanización juega un papel destacado.

\section{Eje 3: Quién ha revelado el diagnóstico}

Con respecto a la categoría sobre quién ha revelado el diagnóstico al paciente, la literatura muestra que el diagnóstico de una enfermedad debe ser dado por el médico responsable, pues él es la persona con la formación necesaria para prescribir el tratamiento ${ }^{(3,4)}$. A pesar de que, en general, es eso lo que ocurre, en algunas situaciones es otro profesional quien revela el diagnóstico, o es un médico sin relación con el paciente, lo que puede levantar dudas, sospechas y dificultades por parte del enfermo a la hora de entender el camino a seguir en su tratamiento.

En la subcategoría Médico, se observó que, en los dos países, quien revela el diagnóstico es el médico de familia o el radiólogo, y posteriormente el paciente es derivado al oncólogo.

"Quien ha descubierto en realidad fue el médico que me hizo la ecografia, la del abdomen total" (Paciente 3 - Brasil).

"Fui al médico de cabecera de toda la vida y me mandó una mamografía. Me lo dijo el patólogo que había visto dos bultos y que era posible que me pasara esto." (Paciente 5- España)

"Me lo dijeron estando ingresada. Primero habló conmigo el ginecólogo y después el cirujano, que fueron los que me operaron. Me lo comunicaron ellos dos." (Paciente 7- España)

En algunos casos se aprecia la insatisfacción de los pacientes españoles a la hora de recibir el diagnóstico por profesionales no especialistas. Los enfermos españoles creen que la manera ideal sería recibir el diagnóstico del oncólogo, pues él es el médico más capacitado para comprender, tratar y diagnosticar el cáncer, y el que aporta mayor seguridad y confianza al revelar el diagnóstico. Por otro lado, los pacientes brasileños no mostraron en su discurso ninguna preferencia por ser diagnosticados por un médico especialista en oncología o por otro médico conocido por ellos.

"Lo ideal sería que el oncólogo sea el encargado de decírtelo no que el paciente llegue a él después de saberlo." (Paciente 11- España)

"Lo idead sería que fuera el oncólogo el que te lo dijera." (Paciente 12- España)

Sin embargo también debe considerarse que la derivación al oncólogo sin previa comunicación sobre la posibilidad de tener un tumor, podría aumentar aún más las sospechas y la ansiedad en el paciente, durante todo el tiempo que debe esperar a 
la cita con el oncólogo. Concretamente en el caso de un paciente español expresó su preferencia por recibir el diagnóstico de cáncer por un médico conocido.

"Me lo dijo el médico de anatomía patológica. Hubiera preferido que me lo dijera un médico más conocido por mí, por ejemplo mi ginecóloga, y de forma un poco más humana, más cercana y tranquilizadora." (Paciente 10-España)

Lo que sí es importante en todos los casos es que el paciente reciba la noticia a través de una persona que le aporte confianza y le haga sentir cómodo para comprender mejor la información que está recibiendo.

En la categoría Familia, el paciente descubre su diagnóstico a través de alguien de su familia. El médico, contrariando las orientaciones de su clase (28), comunica el cáncer al familiar y no al paciente. En esa categoría solo aparecieron testimonios de pacientes brasileños.

"El diagnóstico me ha sido revelado por mi mujer ... Entonces ella vino y me dijo: 'Vamos a la cafetería comer algo'. Allá, ella me dijo: 'Tú tienes cáncer'. Fue un golpe. Yo creo que sería mejor si el médico me diera la noticia. El médico está más preparado para eso que cualquier otra persona." (Paciente 5 -Brasil).

En este aspecto debe tenerse en cuenta que la familia no está preparada para comunicar una información semejante. Si el médico no comunica directamente al paciente la noticia podemos estar ante un problema ético, pues la persona tiene que ser informada sobre su estado de salud. Hace muchos años, se creía que no se debería comunicar la verdad al paciente, debido a la ineficacia y escasez de los tratamientos, por lo que sería peor para el enfermo saberlo. Sin embargo, hoy la tendencia es la opuesta: el paciente debe y tiene el derecho de saber sobre su enfermedad para decidir sobre su tratamiento y el camino que quiere seguir en adelante.

En la subcategoría Descubrió solo, el paciente descubre el mismo que tiene su cáncer, normalmente porque lo lee en el resultado de alguna de las pruebas clínicas realizadas.

"Para mi no ha sido una sorpresa porque yo ya sospechaba, ya habia realizado mi 'autodiagnóstico', sabía que tenía algo..." (Paciente 12- Brasil).

“... ya estaba escrito lo que era (en el examen)” (Paciente 9- Brasil).

"Para ser honesto, yo ya estaba muy desconfiado de lo que era, pues yo vi un examen" (Paciente 14- Brasil).

"Me enteré yo sola al recoger los resultados de las pruebas." (Paciente 11 España)

"Me enteré yo sola al recoger los resultados de las pruebas." (Paciente 11 España)

En casos como los descritos arriba, cuando el paciente descubre solo su diagnóstico, es un momento de grande estrés en el que la persona no va a disponer del apoyo y las explicaciones sobre el diagnóstico que puede aportar el médico. Descubrir el diagnostico uno mismo puede afectarle emocionalmente. 


\section{Consideraciones finales}

La investigación buscó comprender como se produce la revelación del diagnóstico de cáncer a partir de la experiencia de pacientes brasileños y españoles observándose algunas particularidades mencionadas por los pacientes de los dos países. En Brasil aún ocurre que el enfermo puede ser derivado a un oncólogo u otro médico especialista sin una explicación satisfactoria previa sobre lo que puede suceder. También, aunque en los dos países existan relatos de comunicación de diagnóstico en el contexto apropiado (consulta médica), también ha aparecido revelación de manera informal (por teléfono) sin un ambiente adecuado para que el paciente sea acogido y tenga posibilidad de expresar sus miedos y sus dudas. Eso puede expresar una falta de habilidad y de educación en salud para tratar adecuadamente a este tipo de pacientes.

Entre las limitaciones del trabajo presentado podemos señalar el hecho de que los datos fueron recogidos en solamente dos hospitales (uno en Brasil y otro en España), y el contenido es basado solamente en la percepción del paciente y no del médico. Sin embargo, se entiende que los datos mostrados aquí son importantes para comprender que las necesidades de comunicación y de apoyo durante la revelación del diagnóstico del cáncer son semejantes en los pacientes de los dos países, a pesar de las diferencias culturales. Por lo tanto, es necesario que futuras investigaciones examinen cómo está la capacitación para la revelación del diagnóstico entre los médicos para que se puedan desarrollar protocolos de actuación de cara a su capacitación para la comunicación del diagnóstico.

\section{Referencias bibliográficas}

1. Baile WF. SPIKES--A Six-Step Protocol for Delivering Bad News: Application to the Patient with Cancer. Oncologist [Internet]. 2000;5:302-11. doi: 10.1634/ theoncologist.5-4-302

2. Rose C, Stovall E, Ganz P, Desch C, Hewitt M. Cancer Quality Alliance: Blueprint for a better cancer care system. CA Cancer J Clin 2008: 58;266-92. doi: 10.3322/CA.2008.001

3. Hack TF, Degner LF, Parker PA, Baile W, Brundage M, Bultz B, et al. The communication goals and needs of cancer patients: A review. Psychooncology 2005; 14:831-45. doi: 10.1002/pon.949

4. Romeiro FB, Peuker AC, Bianchini D, Castro EK de. Chemotherapy patient perception regarding communication with the healthcare staff. Psicooncologia 2016;13:139-50. doi: 10.5209/rev_PSIC.2016.v13.n1.52493

5. Wright EB. Doctors' communication of trust, care, and respect in breast cancer: qualitative study. BMJ 2004;328:864-0. doi: 10.1136/bmj.38046.771308.7C

6. Kazimierczak KA, Skea ZC, Dixon-Woods M, Entwistle VA, Feldman-Stewart D, N'Dow JMO, et al. Provision of cancer information as a "support for navigating the knowledge landscape": Findings from a critical interpretive literature synthesis. Eur J Oncol Nurs 2013: 17:360-9. doi: 10.1016/j.ejon.2012.10.002.

7. Nelissen S, Beullens K, Lemal M, Van den Bulck J. Fear of cancer is associated with cancer information seeking, scanning and avoiding: A cross-sectional study among cancer diagnosed and non-diagnosed individuals. Health Info Libr J 2015;32:107-19. doi: 10.1111/hir.12100. 
8. Attai DJ, Hampton R, Staley AC, Borgert A, Landercasper J. What do patients prefer? Understanding patient perspectives on receiving a new breast cancer diagnosis. Ann Surg Oncol 2016;23:3182-9. doi: 10.1245/s10434-016-5312-2.

9. Navarro E, Limonero JT, Maté J, Gimenez-Romero M. Necesidades de comunicación e información en el paciente oncológico superviviente. Psicooncología 2010;7:127-41.

10. García ALG, Hurtado AG, Aranda BE. Eficacia de la terapia cognitivo conductual en mujeres con cáncer de mama. Psicooncología 2015;12:129-40. doi: 10.5209/rev_ PSIC.2015.v12.n1.48908

11. Paim J, Travassos C,Almeida C, Bahia L, Macinko J. O sistema de saúde brasileiro: história, avanços e desafios. Lancet 2011;6736:60054-8. doi: 10.1016/S0140-6736(11)60054-8

12. Spiegel W, Zidek T, Karlic H, Maier M, Vutuc C, Isak K, et al. Cancer patients' perception of information exchange between hospital-based doctors and their general practitioners. J Eval Clin Pract 2010;16:1309-13. doi: 10.1111/j.1365-2753.2009.01333.x

13. Cromes GF Jr. Implementation of interdisciplinary cancer rehabilitation. Rehabil Couns Bull. 1978;21:230-7.

14. Eades M, Murphy J, Carney S, Amdouni S, Lemoignan J, Jelowicki M, et al. Effect of an interdisciplinary rehabilitation program on quality of life in patients with head and neck cancer: Review of clinical experience. Head Neck 2013;35:343-9. doi: 10.1002/ hed.22972.

15. Scannavino CSS, Sorato DB, Lima MP, Franco AHJ, Martins MP, Júnior JCM, et al. Psico-Oncologia: Atuação do psicólogo no hospital de câncer de Barretos. Psicol Usp. 2013;24:35-53. [Acceso 10 de enero de 2018] Disponible en: http://www.scielo.br/pdf/ pusp/v24n1/v24n1a03.pdf

16. Arrieira ICDO. A espiritualidade no processo de trabalho de uma equipe interdisciplinar que atua em cuidados paliativos. [Pelotas]: [Thesis]. Universidade Federal de Pelotas; 2009.

17. Vergara AI, Balluerka N. Metodología en la investigación transcultural: perspectivas actuales. Psicothema 2000;12:557-62.

18. Fontanella BJB, Luchesi BM, Saidel MGB, Ricas J, Turato ER, Melo DG. Amostragem em pesquisas qualitativas: proposta de procedimentos para constatar saturação teórica. Cad Saude Publica 2011;27:389-94.

19. Tong A, Sainsbury P, Craig J. Consolidated criteria for reporting qualitative research (COREQ): a 32-item checklist for interviews and focus groups. Inter J Qual Health Care 2007;19: 349-357 doi.org/10.1093/intqhc/mzm042

20. Hounsgaard L, Augustussen M, Møller H, Bradley SK, Møller S. Women's perspectives on illness when being screened for cervical cancer. Int $\mathrm{J}$ Circumpolar Health 2013;72(SUPPL.1):1-7.

21. Perroca MG, Gaidzinski RR. Avaliando a confiabilidade interavaliadores de um instrumento para classificação de pacientes--coeficiente kappa. Rev da Esc Enferm da USP 2003;37:72-80.

22. Clayton JM, Butow PN, Arnold RM, Tattersall MHN. Fostering coping and nurturing hope when discussing the future with terminally ill cancer patients and their caregivers. Cancer 2005;103:1965-75. doi: 10.1002/cncr.21011

23. Fujimori M, Uchitomi Y. Preferences of cancer patients regarding communication of bad news: A systematic literature review. Jpn J Clin Oncol 2009;39:201-16. doi: 10.1093/ jjco/hyn159 
24. Sánchez N, Sirgo A, Hollenstein MF, León C, Lacorte TM, Salamero M. Preferencias de comunicación y apoyo de pacientes oncológicos españoles. Adaptación del "Measure of Patient's Preferences". Psicooncología 2005;2:81-90.

25. Victorino A, Nisenbaum E, Gibello J, Bastos M, Andreoli P. Como comunicar más notícias: revisão bibliográfica. Revista da SBPH. 2007;10:53-63.

26. Skea ZC, Maclennan SJ, Entwistle VA, Dow JN'. Communicating good care: A qualitative study of what people with urological cancer value in interactions with health care providers. Eur J Oncol Nurs 2014;18:35-40.

27. Neto JAC, Sirimarco MT, Cândido TC, Bicalho TC, Matos B de O, Berbert GH, et al. Health professionals and the delivery bad news: patient perspectives. Rev Méd Minas Gerais 2013;23:518-25.

28. Gomes CHR, Silva PV, Mota FF. Comunicação do diagnóstico de câncer: análise do comportamento médico. Rev Bras Cancerol 2009;55:139-43. 